\title{
TaskForce Tarmed SGUM
}

In letzter Zeit wurden einige Krankenkassen wieder aktiver und forderten von mehreren Ärzten teilweise namhafte Beträge zurück wegen angeblich unzulässiger Verrechnung von Leistungen ohne Berechtigung oder Nichtbeachtung von Kumulationsverboten. Diese Rückforderungen betrafen sogar auch Vorjahre.

Während ein Teil der Rückforderungen durchaus berechtigt waren, mussten wir aber doch wegen oft vorhandener mangelhafter Tarifkenntnissen von Kassenmitarbeitern einerseits aber auch von betroffenen Ärzten andererseits einschreiten. An dieser Stelle möchte ich nochmals auf die Internetseite www.arzttarif.ch hinweisen, auf welcher viele Informationen betreffend die Abrechnungen aus dem Kapitel 39 (Bildgebende Verfahren) aufgeschaltet sind.

In Sachen „Verrechnung der Gegenseite bei seitenvergleichenden Extremitätenaufnahmen" ist die gross angelegte Multicenter-Studie angelaufen. Während zweier Monate werden an 22 Zentren in der Schweiz alle routinemässigen UltraschallUntersuchungen ausgewertet. Die Untersucher sind allesamt entweder Tutoren oder Kursleiter, damit die Qualität der Untersuchungen möglichst optimal ist und die damit verbundenen Fragestellungen zu einem relevanten Ergebnis führen. Die Resultate werden im Herbst 2012 vorgestellt und publiziert.

Beat Dubs, Leiter TaskForce Tarmed SGUM 